\title{
How Cotton Fibers Become Yarn
}

\author{
Experiments unravel the mysterious twisting process by which short \\ fibers bind together into yarn.
}

\section{By Michael Schirber}

$\Lambda$ strand of cotton or wool yarn is a long chain of short fibers held together not by knots or glue but by the simple twisting of fibers around each other. New experiments explore how twisting changes fibers from a loose collection of independent strands into a single strand of yarn that is hard to break [1]. The researchers show that twisting increases the fiber-to-fiber binding strength up to a critical amount of twist, above which the binding strength is limited by the breaking of fibers. The analysis reveals an optimal fiber radius, which might be useful for designing environmentally friendly yarns that mix cotton with other fibers.

Yarn technology is old; evidence of twisted fibers has been found at many prehistoric sites, including one from 40,000 years ago [2]. The traditional technique for making yarn, called spinning, starts with thin fibers of, say, flax, that are up to a few tens of centimeters long. A tangled collection of these fibers is stretched and twisted to make a long, narrow string composed of overlapping fibers bound together only by twisting around each other. Over the centuries, many scientists-including Galileo-have been intrigued by this binding of fibers, but a

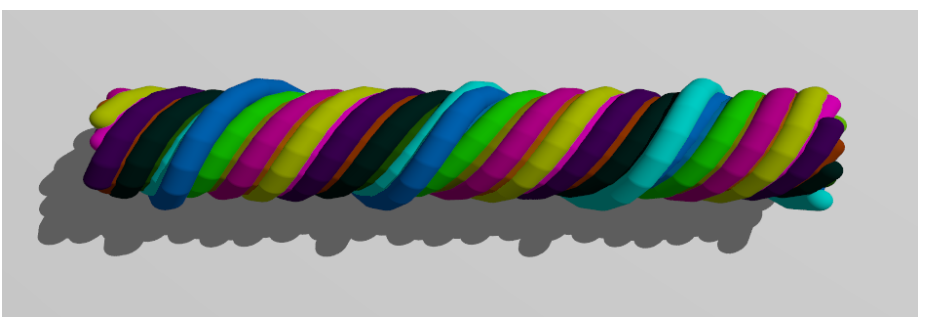

An added twist. Like real yarns of cotton or wool, the more these simulated fibers are twisted, the harder it becomes to pull the virtual yarn apart.

Credit: J. Crassous/Univ. of Rennes model describing the twisting process has been lacking, says Jérôme Crassous from the University of Rennes in France.

Crassous and Antoine Seguin from the University of Paris-Saclay measured the binding strength in fibers as the amount of twisting was incrementally increased. They started with sets of cotton or flax fibers knotted together at one end-like the tassel on a college graduate's cap. They overlayed two of these tassels with their knots protruding in opposite directions and twisted them so that their loose ends became interlaced together. As in normal yarn, the twisting increased the contact area between the fibers, resulting in a larger frictional force. To determine this binding force, the duo pulled the knots in opposite directions and measured the pulling force at which the two tassels began to slide apart (see Synopsis: The Strength of Interleaved Phonebooks Explained).

The researchers found that the binding strength increases rapidly with the number of turns. "Initially, the fibers separate easily," Crassous says. "But then you turn just once more, and it becomes nearly impossible to separate them." For example, the binding force for $80-\mathrm{cm}$-long cotton fibers was around 200 newtons (N) after 8 turns, but this force jumped to $500 \mathrm{~N}$ after 9 turns. The researchers found that adding more turns than 9 didn't help, as the fibers began to break for applied forces greater than $500 \mathrm{~N}$.

Crassous and Seguin obtained similar results for a wide variety of cotton and flax fibers of various lengths between 10 and 80 $\mathrm{cm}$, as well as for numerically simulated fibers. They showed that all of their data could be described using a parameter they call the Hercules twist number, which combines the twisting angle with relevant fiber properties, such as the friction coefficient and the radius. For all of the yarns tested, this number-proportional to the square of the number of 


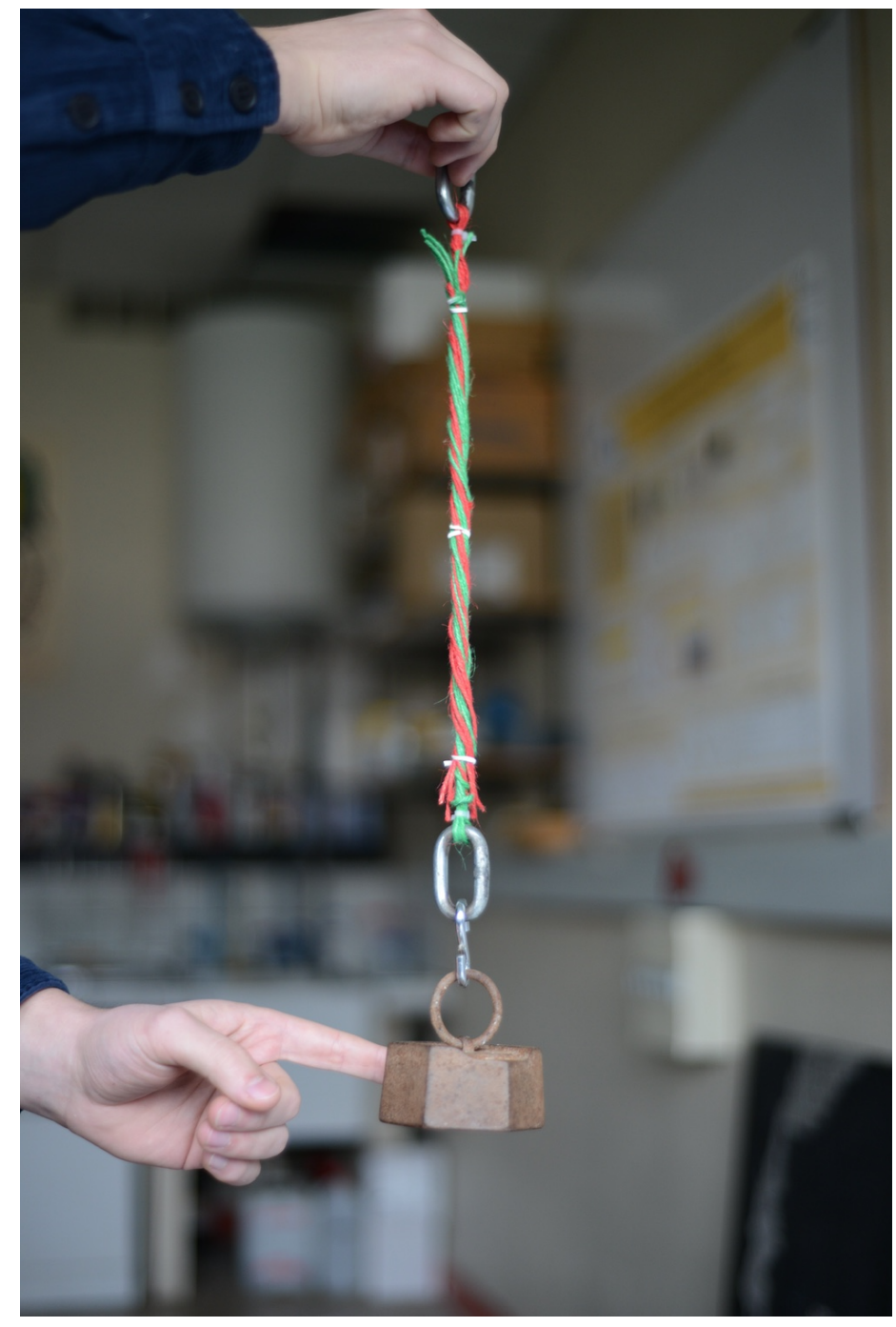

Holding on. Researchers overlay two tassel-like strands of fibers (one green, the other red) and twist them together. That interlacing alone is able to keep the strands from sliding apart when a weight is applied to one end. (The white strings were used to align the fibers before twisting, but their effect on the sliding resistance is negligible, the researchers say.)

Credit: J. Crassous/Univ. of Rennes twists-had a critical value of 30. This amount of twist provided enough friction that the strength of individual fibers became the "weakest link," and the yarns would simply break when pulled, rather than unraveling. Using this critical twist value, the researchers determined the fiber radius that produces the strongest yarn for a given material. For cotton, this optimal radius is 80 micrometers, which agrees with what is typically used for cotton yarn. Yarn made from thicker fibers would be weaker.

Crassous admits that optimizing strength isn't necessary for cotton yarn and hemp ropes, which have had thousands of years to be perfected. However, some environmentalists are advocating for mixing cotton fibers with more environmentally friendly fibers, but there is still some uncertainty about how to maximize the strength of these blends. Crassous thinks that his work with Seguin could provide insights.

Materials scientist Patrick Warren from the Science and Technology Facilities Council in the UK says that the researchers have developed a clean experimental model for spun yarns, which has allowed them to tease out a general formula with the Hercules twist number. "This is then an important addition to the relatively small but growing literature exploring the statistical physics of mechanical assemblies of frictional objects," Warren says. He thinks that further experiments could explore the friction forces in splices, which are entangled-fiber junctions used to join separate pieces of rope or yarn.

Michael Schirber is a Corresponding Editor for Physics based in Lyon, France.

\section{REFERENCES}

1. A. Seguin and J. Crassous, "Twist-controlled force amplification and spinning tension transition in yarn," Phys. Rev. Lett. 128, 078002 (2022).

2. B. L. Hardy et al., "Direct evidence of Neanderthal fibre technology and its cognitive and behavioral implications," Sci. Rep. 10 (2020). 